\title{
2.
}

\section{Character Diversity in Digital and Non-Digital Games}

Alexandra To, Joselyn McDonald, Jarrek Holmes, Geoff Kaufman, \& Jessica Hammer

Transactions of the Digital Games Research Association August 2018, Vol 4 No 1, pp 31-65

ISSN 2328-9422

(C) The text of this work is licensed under a Creative Commons Attribution -- NonCommercial --NonDerivative 4.0 License (http://creativecommons.org/licenses/by-nc-nd/ 2.5/).

IMAGES: All images appearing in this work are property of the respective copyright owners, and are not released into the Creative Commons. The respective owners reserve all rights.

\section{ABSTRACT}

We explore how digital and non-digital games express diversity through characters, understood as representations of marginalized groups to which the player may or may not belong. We identify a range of techniques using visual design, abstract character representation, delineation of game setting and roles, conversation design, and the design of rules and systems. We illustrate these 
techniques with exemplar games identified through consultation with experts. This analysis yields four key recommendations for designing diversity across a range of game platforms: match diversity affordances to player needs; draw strengths from both the digital and non-digital realms; design for conversation; and consider player diversity. We conclude by proposing diversity as an end-to-end process in both game research and design.

Keywords

Diversity, game design, non-digital games, digital games, character design

\section{INTRODUCTION}

Digital and non-digital game designers alike face challenges in representing diversity meaningfully and authentically in games. Looking beyond the most obvious reasons for this persistent dearth of diverse representations - marketability pressures and assumptions about gamer audiences, a lack of diversity among game designers, reversion to previously established norms of stereotypical character depictions - we find that even when designers earnestly attempt to include diverse characters, their efforts often fall short. A major criticism leveled at game designers in this regard concerns their focus on promoting pluralism rather than representing diversity in the narrative worlds they create. Pluralism allows players to interact with characters like themselves, for example by creating avatars that reflect their own identity, while diversity exists when players experience and interact with representations of marginalized groups, including but not limited to groups to which they themselves belong (Shaw 2010).

In practice, representations of diversity in games have focused almost exclusively on a small number of categories such as race and gender (Williams et al. 2009; Shaw 2012; Cole et al. 2017). 
Other identities, such as sexual orientation, age, religion, language, citizenship, and neurodiversity are often not represented in even the most diverse games (ibid.). Further, even when race and gender are represented, the representation typically focuses on physical features (e.g., skin tone) and does not touch on cultural, psychological, or experiential aspects of those identities. Representation is then, almost literally, skin-deep; players do not have access to experiences that surface those other identities or deeper senses of identity. As well, designers have struggled to express non-visible identifiers (like sexual orientation or religion) within a game context without reverting to stereotypes. For example, presenting a romance storyline is often the easiest way to reveal a character's sexual orientation, but, in reality, we know that sexuality holds a much deeper meaning to a person's identity than just their romantic relationships (Shaw 2009). As a result, many game designers simply avoid tagging characters with invisible identifiers (like sexuality or religion) to circumvent the difficulty of incorporating that identity within the game narrative (Shaw 2009).

Queer game studies challenges this status quo by seeking queer representation in ways that go beyond the visual, and in contexts that go beyond the representations of sexuality (Ruberg \& Shaw 2017). Queerness can be encoded in mechanics, in aesthetic experiences, and even in the relationship between the player and the game. While queerness can refer to sexual orientation and/ or gender identity, it also functions as a critical paradigm that challenges normative power dynamics, social orders, and hierarchies (Jagose 1996). As such, the call for queering representation in games can apply to other types of non-normative identities, particularly because queerness and other marginalized identities are not mutually exclusive (Ruberg \& Shaw 2017).

Inspired by queer game theory, the current work pushes back against the notion that representation is encompassed primarily by a character's appearance, particularly when it comes to invisible identities. We therefore explore the question of how game 
designers represent diverse and non-normative character identities in ways that go beyond the visual - a pressing question given the centrality of characters and character-driven narratives in many game genres. Additionally, previous theorists have argued that a game's platform influences the types of mechanics that are possible, what systems can be implemented, and how game assets can be produced (Bogost \& Montfort 2007). This approach suggests that when games represent non-normative identities through the lens of characters, different platforms may afford different possibilities for design and interaction.

In this paper, we solicited expert recommendations to develop a list of digital and non-digital game exemplars that include diversity, understood as non-dominant and/or non-normative character identities. We coded the games for the presence of both visible and invisible identities, conducted game design analyses, and identified specific design techniques being used to represent non-normative identities in novel and compelling ways. We derive five areas in which character diversity can be communicated: 1) visual design, 2) abstract representation, 3) setting and roles, 4) conversation design, and 5) rule and system design. We conclude by examining how the design strategies used by these games are linked to the affordances of their medium, and what larger implications they have for diversity in games.

\section{LITERATURE REVIEW}

To explore how games represent diverse identities, we consider what we mean by identity. We draw from identity theories in a range of fields, including sociology, psychology, and gender studies, to frame identity asrelational, self-relevant, and intersectional- qualities that inform our methods and analyses.

Conceiving identity as relational counters the frequent attribution of identity to aspects of oneself that are immutable, innate, and visible, and instead argues that identity is actually constructed dynamically and socially through a wide variety of interacting 
elements (McCall 2005). Social categories (e.g., soccer team, family) help to define an individual's identity (Hogg et al. 1995), but they also provide a context within which the individual's identity becomes meaningful (Wiley 1991). We therefore consider identity as it is enacted in social contexts rather than as static labels or categories.

Self-relevance is the notion that aspects of one's identity might be more relevant to some than others. Identity scholars argue that individuals construct a hierarchy of identities, with the most relevant being those most likely to lead to action (Hogg et al. 1995; Wiley 1991). The self-relevance of identity features is impacted by a variety of personal, societal, relational, and/or situational factors. In our research context, we recognize that each player embodies a range of identities - some are more likely than others to be reflected in gameplay and may have highly variable degrees of self-relevance.

Intersectionality theory also emphasizes that players occupy multifaceted social positions, and simultaneously experience identity features (Crenshaw 1989; McCall 2005). These multiple identities produce new experiences at their intersection. For example, the intersection of womanhood and blackness produces misogynoir (Bailey 2010), a specific set of disadvantages and stereotypes that are neither like the experiences of black men nor of non-black women. In other words, our identities are greater than the sum of our parts (Crenshaw 1989). This insight motivates our investigation of multiple representations of marginalized identities.

In addition to their implications for identity development and expression, interactions with diverse game characters can help reduce bias and prejudice through several different psychological mechanisms. First, counterstereotypical representations can effectively reduce biases and prejudice by altering players' schemas, or mental representations, of outgroups (Dasgupta \& Greenwald 2001). For players who share group affiliation with 
those characters, these counterstereotypical representations can also provide a psychological buffer against stereotype threat (Marx \& Roman 2002). Second, perspective-taking, or the appreciation for others' unique psychological points of view, has been shown to be facilitated through exposure to the experiences of diverse others (e.g., Davis et al. 1996, Galinsky \& Moskowitz 2000) and includes beneficial outcomes such as greater overlap in mental representations of self and other (e.g., Davis et al. 1996), and decreased stereotypes (e.g., Galinsky \& Moskowitz 2000). Finally, taking on an alternate identity in a fictional context allows players to simulate the subjective experience of that character, a phenomenon known as experience taking. Research with readers showed that when experience-taking occurred with characters belonging to other social groups (such as protagonists of a different race or sexual orientation), it led to lower reported levels of prejudice and stereotyping toward those groups (Kaufman \& Libby 2012).

These theories have a range of implications for game design. For example, exposure to diverse characters builds on notions of parasocial interaction, through which individuals form a pseudorelationship with those characters and imagine them as part of their real-life social sphere (Hoffner 1996), while character-driven narratives appear to encourage deeper levels of empathy when they feature narrators or protagonists that differ in important ways from one's own identity or experience, or that fall outside of one's typical social sphere (Mar et al. 2006; Kidd \& Castano, 2013). Experience-taking theories, on the other hand, ask the player to mentally simulate the experiences of a character who is different from themselves (Kaufman \& Libby 2012). Research has shown that players can temporarily simulate aspects of a target character's identity (Kafai et al. 2010). However, something about the context or process of that simulation must stimulate an authentic, bottomup awareness of difference, one that is uninfluenced or unfettered by their own assumptions or preconceptions. 
The techniques available to designers will vary depending on the different and complementary affordances of digital and non-digital technologies. In this work, we use Faraj and Azad's (2012) definition of affordance as "the enactment of several mutuality relations between the technology artifact and the actor." This definition allows for a given artifact to have multiple contextuallydependent affordances related to a given actor, group of actors, varying focal contexts, etc. We highlight the difference between what we can easily accomplish with digital and non-digital game materials in order to articulate how these differences impact the ability to implement diverse representations in game characters and the ability to represent non-normative or marginalized identities regardless of whether the player shares those identities.

In reviewing digital technologies, we focus on affordances that can be directly juxtaposed with the affordances of non-digital games. First, digital technologies allow for automated processes in response to participation and interaction - users are afforded a wide range of complex interactions which are followed by a set of rule-based procedures and automatic data processing resulting in dynamic environments (Jiow \& Lim 2012, Murray 2011). Second, digital technologies are social - they can encourage, facilitate and even enforce and require social interactions and social participation, as well as surface and make visible both individual and social interactions (ibid). Third, digital technologies allow for the creation and maintenance of multiple identities that have varying mutuality relationships with the offline self (ibid).

Non-digital games can include a wide range of physical elements, from traditional elements such as cards (Altice 2014) to unusual custom elements such as Larklamp, a lantern-based game (Warne 2017). However, most non-digital games share many of the affordances of paper, as outlined in Sellen \& Harper (2004): they occupy physical space, they are hard to replicate, they cannot be remotely accessed, and they display static information. However, they can also be laid out in space as a way of organizing information, navigated flexibly, and annotated or modified using 
ordinary household items. Non-digital games also effectively foster discussion among co-located participants (Kaufman et al. 2016a, Xu et al. 2011).

\section{METHODS}

In our work, we incorporate reflexivity on our own position as critics and writers. Collectively, we have a range of non-normative identities, including race, gender, religion, and sexual orientation. At the same time, we recognize that there are aspects of marginalization that we do not experience. For example, we are all comparatively young, cisgender, and citizens of the country in which we reside. We also recognize that our experiences do not and cannot represent everyone who shares a particular identity. Finally, we recognize that we are, collectively, both scholars and practitioners of game design. This social position informs how we analyze games as well as how we frame the implications of our work. We hope to speak specifically to communities that we belong to, namely game designers and game researchers.

To address these issues, we turned to the literature to develop a list of the qualities, attributes, demographics, and behaviors (social and others) that inform identity, rather than relying on our own experiences. The non-exhaustive list of features we used to guide this inquiry were: body diversity, citizenship, culture, gender, generation, neurodiversity, physical ability, race, religion, sexual orientation, and socioeconomic status. We then recruited experts to help us develop a list of exemplary digital and non-digital games that address diverse character representations. To generate this list, we contacted 16 game scholars and designers from both academia and industry. Based on our list of features, we requested recommendations of games that thoughtfully incorporated diverse identities and perspectives, or that explored, challenged, and subverted normative identities. We then narrowed the selection to games with narrative elements and human or human-like 
characters. Appendix A lists the games remaining in our list after this narrowing process.

Next, members of the research team were randomly assigned games to analyze. For each game, the team member drew on a range of reference materials, including their own gameplay (Bizzocchi \& Tanenbaum 2011) as well as gameplay videos, fan sites, and promotional materials. The game was coded for the presence of a range of identity factors, as per the list of features above. Once coding was complete, team members selected games to analyze more fully, either through further consultation with experts or by using public rankings such as Board Game Geek's Top 1000 rated titles. Analysis was conducted using techniques drawn from Fernández-Vara (2014), Ruberg \& Shaw (2017) and others. In particular, for each identity axis present based on the coding scheme, team members identified and documented design decisions in which that identity was instantiated.

The team then collaboratively reviewed the resulting documentation and discussed themes that emerged across games. The team used qualitative research methods (e.g., Strauss \& Corbin 1998) to code, organize, and discuss the material along a range of axes, including genre (e.g. comparing techniques within board games), identity type (e.g. different ways of representing gender), and approach to identity representation (e.g. giving the player control). During this iterative coding process, the team noted that non-normative character identities were represented within the game in five different ways. We present our findings based on these themes.

\section{CHARACTER DESIGN APPROACHES}

Five representational strategies for character identity emerged from our design analyses. We identified strategies for visual design of characters, particularly those that differed from playercontrolled avatar customization; for representing internal or abstract elements of a character, such as skills or traits; for 
character roles within a game setting, and how those roles convey identity; for the design of conversations between or about characters; and for designing rule systems that define characters' capacities in action.

For each of these five types of representation, we present both digital and non-digital examples. We also describe similarities and differences we observed in between the strategies used in digital and non-digital games. Because our game sampling technique relied on experts choosing exemplary game titles rather than on an exhaustive search, we recognize that these analyses are illustrative. Additionally, we do not attempt to present all design decisions found in our game sample; we choose examples that are representative of larger patterns.

Finally, our five modes of character identity representation are not meant to be mutually exclusive - rather, the reverse. Design decisions made around representing non-normative character identities are often interdependent. For example, a game's strategy for representing abstract elements of a character is likely to affect the design of rules that interact with those elements, and vice versa. We have deliberately chosen examples that illuminate some of these relationships.

\section{Visual Design}

As suggested by prior work in this area, the visual design of characters emerged as one method for marking identity (Cole et al. 2017). Avatar customization is currently a dominant strategy for addressing identity; digital character customization mechanisms are being studied for their impact on players (Turkay \& Adinolf 2010), and also receive critical attention for their limitations and failures (McArthur et al. 2015). These methods were present in a number of games in our study, such as the Dragon Age series. However, we observed that approaches other than user-controlled customization could be used in diversity-supporting ways. 
ToDiGRA 41

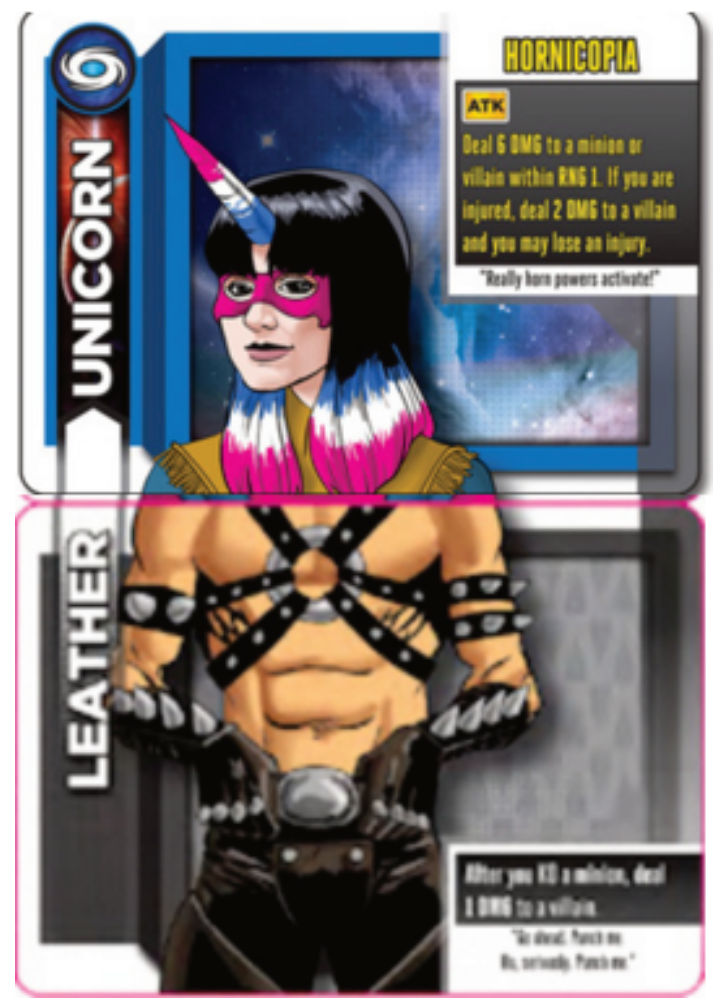

Figure 1. The "unicorn" card reads more typically feminine, while the "leather" card reads more typically masculine - the two are combined to create a hero.

Heroes Wanted (Chance \& Little 2014) uses randomized character creation to disrupt player assumptions and challenge norms, particularly about gender. Players are dealt three cards each for the top and bottom "halves" of the characters, with a balance of attributes and illustrations for various gender identities, ethnicities, body types, etc., as well as elements that represent more masculine and feminine traits or elements (e.g., leather costume versus pink hair/makeup). Moreover, the character element cards contain a fair number of gender stereotype-defying exemplars (e.g., a female cop, cobra, and ninja), and the character illustrations are also, in some cases, ambiguous (e.g., gender-ambiguous faces such as the one for the "rainbow" character card). Because characters are 
made from multiple cards, the game can produce unusual and unexpected combinations (e.g., a leather unicorn hero (Figure 1)) that challenge norms and, moreover, take the form of associative links between categories and attributes that correspond to the ways that stereotypes are cognitively represented; thus, through exposure to an array of attribute combinations in "completed" heroes, players have the opportunity to experience (and embody) heroic, yet relatable, characters who may challenge preconceptions (e.g., "fancy beard” or "heavy metal ninja”). The versatility that the sheer volume of covarying traits that this system of character creation combinatorics affords effectively, reinforces the complex and multi-faceted nature of personal identity.

Although games in the Dragon Age series allow the player to generate their own customized avatar, the other characters in the game are not customizable by the player. This allows the designers to question norms that players might otherwise replicate. For example, the designers question norms around age by filling the world with both major and minor elderly characters, such as Leandra Amell, the protagonist's mother; Wynne, a powerful mage; and Meredith, an antagonist and military leader. Because there are multiple older characters present both within individual games and across the series, the diversity among those characters forces the player to question whether their assumptions about the elderly are correct. The game also tackles stereotypes head-on through the character of Flemeth (Toma 2015). Flemeth is initially portrayed as a wrinkled woman with graying hair and a frail body, and often refers to the player as "child." However, in Dragon Age: Origins (BioWare 2009) she shapeshifts into a powerful dragon, and in Dragon Age II (BioWare 2011) she is shown as a warrior. While the player can predict these changes, particularly if they have played the game multiple times or consumed media about the game, the player's character is always fooled by Flemeth's stereotypical behavior. This creates an ironic distance between player and character that allows the player to reflect on their own assumptions about age. 
Detailed customization for visually depicted avatars is not typically available in non-digital games (though some types of non-visual customization are well-explored; see below). However, the strategy of reducing player agency around visual markers of identity is available in both digital and non-digital game contexts. Based on the examples in our sample, this strategy relies on visually inclusive elements created by the designers, such as: elderly character models or cards bearing ambiguous gender markers; multiple exemplars of the identity in question, which can occur within a single game, across multiple play sessions, or across multiple games in a series; and awareness of likely player assumptions about the identity axis in question (e.g., that old women are helpless or that gender is fixed and uniform).

\section{Abstract Representation}

Avatars, portraits, and other visual representations are not the only ways that characters are encoded in games. Characters can also be represented with non-visual elements, such as character statistics. The elements can be defined by visual elements of the character, such as Heroes Wanted linking character drawings to underlying attributes by placing them on the same physical card. However, they can also exist in games without defined visual representation for characters, or can vary independently of how a character looks, as in our examples below.

Long Live the Queen (Hanako Games 2012) has a consistent visual representation for its main character, Princess Elodie: a pinkhaired, large-eyed fourteen-year-old girl. However, Princess Elodie changes over the course of the game in other ways. The player must teach Princess Elodie the skills she needs to become a queen, which are represented numerically and abstractly (Figure 2). Elodie's skills are listed on the skills screen; a higher score means she is more capable in that area. The list includes both typically feminine skills (e.g., elegance, decoration, dance) and ones that are often associated with masculine roles (e.g., swords, naval strategy, accounting). However, despite Elodie's 
conventionally feminine appearance, the game treats all skills as equally valuable, and she begins the game equally ignorant of all of them. The game is making two clear statements. First, Elodie is not limited to feminine traits simply because she is a princess. Second, Elodie does not need to reject feminine traits in order to become a successful leader. Rather, the player must pay careful attention to the challenges that Elodie faces, and draw strengths from across stereotypically feminine and masculine traits to meet them.

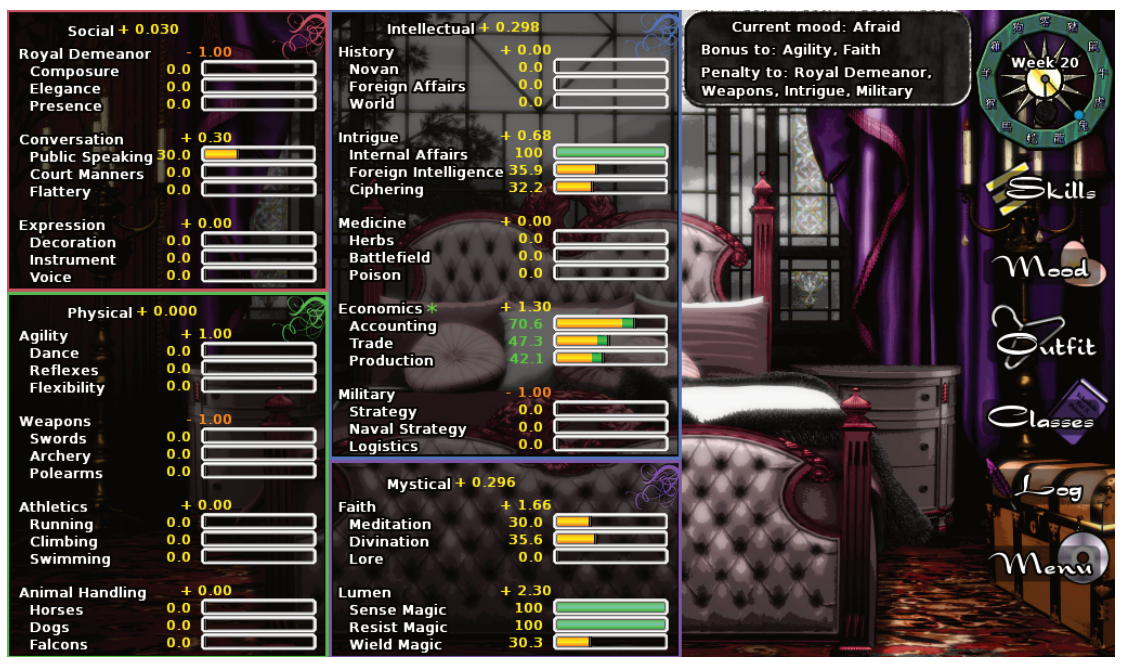

Figure 2. Elodie's skills are broadly categorized as social, physical, intellectual, or mystical, with a variety of sub-categories and skills.

Games such as Legends of Andor (KOSMOS 2012) and Robinson Crusoe: Adventures on the Cursed Island (Portal Games 2012) use precisely the reverse strategy: keeping internal statistics the same no matter whether the character is visually depicted as "male" or "female." While the intention here is ostensibly to allow players to make their own personal choice, the implicit message is one of equivalence, that there is essentially no difference in intelligence, fortitude, or power between the male and female instantiations of the character. In a world that treats men and women differently, games that maintain traits, attributes, and abilities (particularly ones that are often seen as gendered) regardless of character 
gender are making a strong statement about male and female capacities.

In the tabletop role-playing game Steal Away Jordan (Ellingboe 2007), characters are designed by players, but are not represented visually. Instead, players describe the character's history and social situation, which are then translated into abstract game statistics. The latter is particularly important, as the game is set in the antebellum American South, and most players take the role of slaves. Every character has a statistic called "Worth," which reflects how valuable they are to the society in which they live. To drive home the socially defined nature of worthiness, a character's Worth is defined by the gamemaster and not by the player who controls that character during play. A full four pages of the game's rulebook are dedicated to explaining factors that the gamemaster should take into account when assigning Worth, such as age, sex, special skills, injury or disability, literacy, and a slave's relationship with the slave owner and his family. Players with slave characters are explicitly instructed that they can increase their character's Worth by taking the master's interests as their own, for example by keeping other slaves in line. This representation asks the player to engage not just with African-American history, but with how normative and non-normative identities are socially defined.

Both the digital and non-digital games in our sample made strong statements based on the structure of their abstract representation of characters, either through what was included in the representation (e.g., the incorporation of elegance in Elodie's skill list, or the nature of Worth) or through how elements of the representation related to one another (e.g. introducing no underlying differences between male and female characters). However, we observed differences in how these representations changed over time. The non-digital games had flexibility in how game statistics were updated. For example, in Steal Away Jordan, players are given examples of how they can raise their character's Worth, but are expected to improvise and innovate in line with those examples. 
The digital games enabled complex updates to character representations; for example, determining how quickly Elodie improves her skills involves a long series of complicated computations, which has to be repeatedly executed during play. While it might be technically possible for human players to compute Elodie's skill improvement on their own, offloading the computation to the computer makes the game playable - and mastering the complexity of this mathematical system is a core element of play.

\section{Setting and Roles}

In narrative games, characters exist in a fictional context, and typically have social roles within that context. Both a game's setting and roles can cue players about how to behave (Baldwin 1992; Markus \& Wurf 1987). Some games do substantial secondary world-building to create a fictional context for the characters, such as the Dragon Age series which features in-game books full of legends and lore. Others rely on common tropes and use the player's prior knowledge to fill in details of the setting. For example, Dead of Winter: The Long Night (Plaid Hat Games 2016) uses social roles, such as "doctor," to communicate a character's abilities and role in the game, which otherwise has a very lightweight setting.

Some games use their setting to first establish, and then defy, normative power structures around character identity. For example, when playing elven or dwarven characters in Dragon Age: Origins, players can be the target of, respectively, racial and caste-based bias. These biases are embodied in interactions with non-player characters, such as slurs directed at a dwarven player character by higher-ranking dwarves. Power structures are also reified in the physical layout of the world, such as placing Dalish encampments physically far from locations of wealth and power. The game then challenges these power structures by placing the player character at the center of the game's story. No matter how 
the non-player characters treat them, only the player's character can ever save the day.

Dream Daddy (Game Grumps 2017) does the reverse, subverting norms by imagining a world where all the adult men in a suburban neighborhood can, and do, date one another. The game treats their sexual orientation as normative by using it as the unquestioned backdrop for the game's activities. The player's concerns are choosing which hot dad to date and making decisions that can determine if the dates go well; even the game's jokes rely on "dad" humor and stereotypes, not stereotypes about gay or bi men. Simultaneously, the player must ensure that his character's daughter ends up successfully navigating the transition between high school and college, subverting the typical assignment of caring work and relationship management to women. To enter into the spirit of the game (Suits 2014), the player must accept these premises.

In Monsterhearts (Alder 2012), players take on the role of literal monsters, such as vampires and werewolves who attend high school. The characters' monstrous, non-normative identities stand in for "experiences of alienation, shame, queerness, and selfdestruction," while the high school setting creates a constrained, oppressive social structure within which the characters must survive. However, players must co-create the details both of the dangerous, "feral" world of their characters, and the normative society that treats them as monsters. To balance these goals, players are asked to produce physical game aids that are rough, messy, ambiguous, and partial, but that also provide structure for gameplay. For example, the players collaboratively create a seating chart for the characters' homeroom. Players decide where their characters sit, then begin to define facets of identity for other characters who sit nearby. In this collective process the MC is directed to sketch roughly and to take only those notes that support play. By taking the time to generate a physical-but-imperfect representation of the shared character and setting knowledge, the 
players come to understand ambiguity as the space where their characters can survive.

When engaging with non-normative or marginalized identities through a game's setting or roles, the game must find ways to undermine players' prior assumptions. We observed that games did this in two ways. First, some games piggybacked the question of non-normative identities onto game material about which the player would have limited prior knowledge, such as the social structures of elves and dwarves, forcing the player to learn the game's framing. Second, some games inserted non-normative identities into strong existing tropes, such as high school drama, then relied on the player's use of those tropes to explore those identities in interesting ways. In digital games, these strategies could be accomplished through content delivery. Non-digital games, particularly role-playing games, sometimes attempt to deliver large quantities of setting material (e.g. in the form of game books), but struggle with players internalizing and using that knowledge. On the other hand, non-digital games more easily allow players to co-create the game's setting; players must internalize the setting to a greater degree in order to use it in play, since the game does not digitally enforce adherence to the game's social norms. Players may also need to discuss the game in order to agree on how the setting should function, producing interesting conversations.

\section{Conversation Design}

In our sample of games, we observed that many games with characters used diegetic conversation, or conversations occurring within the frame of the game, to help define character identity elements. Pre-scripted dialogue types included dialogue between computer-controlled characters, dialogue directed at playercontrolled characters, and dialogue controlled by the player, while role-played conversations involved players improvisationally speaking in the roles of characters. Other games incorporated meta-conversational techniques, methods for starting player-to- 
player conversations about the identities of the characters in the game. While these conversations are outside the scope of the game itself, game design decisions can either foster (Xu et al. 2011) or undermine (Khaled 2014) reflective conversations between players.

Many diegetic conversations in Dragon Age feature the PC's companions, computer-controlled characters who accompany the PC throughout the game. The game incentivizes the player to talk to their companions because conversations with companions can unlock side quests and romantic options, but companions also allow the game to portray marginalized identities. For example, in $D A: I n$, the companion, Krem, is a transgender man, which is only revealed after the player has initiated many conversations with Krem and earned the character's trust. Because the identity is brought up in conversation, Krem can share the internality of his experience rather than relying on visual markers to cue the player.
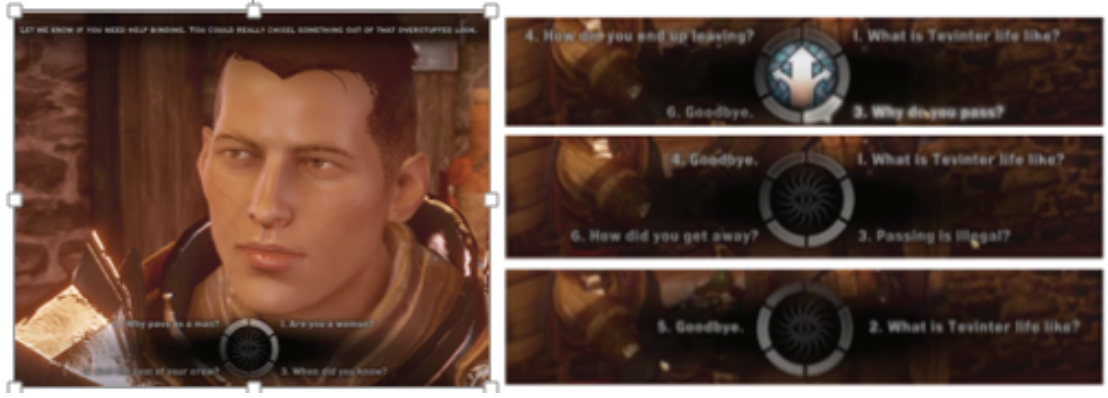

Figure 3.Krem self-discloses his gender identity through a comment about binding. The player can engage further in the dialogue but is restrictedthey can ask probing questions but cannot be aggressive or explicitly transphobic.

Additionally, the conversation design in Dragon Age relies on dialogue trees: during a conversation, the player can select from pre-generated responses, but not generate options of their own (Figure 3). The game uses this to model appropriate behavior; the game does not provide options for many common bigoted responses to trans identity, even if the player may wish to respond that way. Other dialogue options appear questionable (e.g. "Why 
pass as a man?” or “Are you a woman?”), but when the player selects those, the response actually spoken by the character is either softened (e.g., “Are you a woman?” translates to “Oh, are you? I didn't realize...”) or the character will be firmly rebuked (e.g., Krem friendly but sarcastically responds, "Great! Now we can all talk about it!”).

The board game Ladies and Gentlemen (Lamy 2013), on the other hand, generates conversation between players by putting stereotypes front and center, inviting players to confront, subvert or cast a humorous or ironic slant on them. Players are paired off, with one player assuming the role of aggressive bread-winning husband, and their teammate the role of frivolous, dress-shopping wife (who must implore her spouse to provide her sufficient funds to indulge her obsession with fashion and glamour). The game's tongue-in-cheek rules manual exaggerates these overt stereotypical roles, and from both the personal experience of the authors and other players' accounts of the game from its Board Game Geek forum, incites playful roleplay, often involving male and female players assuming the role of the opposite gender. This game and others like it, such as Cards Against Humanity (Dillon et al. 2009), entail a high level of player interactivity in navigating and "playing with" their overt stereotypical content; however, they are correspondingly reliant on player willingness to engage critically.

Adding structure to meta-conversations can help provide a critical framing. For example, the LARP scenario, Against the Grain (Turkington 2016), explores a historical wildcat hate strike by white women working in a Baltimore factory in 1944, who were protesting the first African-American woman to join their crew. Players take on the roles of stakeholders in the conflict, including characters with racist and sexist attitudes, and the game's design pushes players to have conversations as characters that embody racism and sexism. For example, the Bird-in-Ear technique allows the game's facilitator to undermine the marginalized characters, or harden the bad behavior of dominant-group characters, by 
whispering what society thinks of their decisions. However, the game also includes a facilitated post-game debrief where the facilitator asks players to reflect on their game experiences, including explicitly asking about what they will take away from the game and what they hope to leave behind. The diegetic conversations provide the raw material for the meta-conversation about what the game implies for players.

In our sample, digital games primarily relied on pre-scripted dialogue with limited player input. These designs allowed more control over how identity was engaged through conversation, including controlling when identities are revealed, modeling appropriate reactions to diverse identities, and forestalling hateful or bias-reinforcing conversational directions. However, these games included many of the challenges to reflection noted by Khaled (2014), such as a high level of immersion and a quantification of conversational outcomes. Conversely, non-digital games included both improvisational diegetic conversations, when players either formally or informally adopted the role of in-game characters, and techniques to provoke meta-conversation, such as humorous exaggeration and explicit debrief. However, they had less control over the content of conversation, and risked provoking conversations that replicate negative attitudes about diversity, rather than supporting it.

\section{Rule and System Design}

Character identities and game norms can be introduced, reinforced, and expressed through rules that constrain characters' actions, choices, and interactions. These rules typically interact with other aspects of the character defined above, such as representations of their internal states or their in-game roles.

Hellblade: Senua's Sacrifice casts the player as Senua, a warrior seeking to return her beloved to life. Senua experiences psychosis, which is represented in part through rules design. The game's key puzzle mechanic requires finding environmental patterns that 
correspond to a particular rune. Senua's ability to find these patterns is framed as a direct result of her psychosis. By making what might otherwise be framed as “delusions” integral to Senua's progress in the game, the player is encouraged to see her mentally diverse perspective as a strength. At the same time, Senua is tormented by voices that no one else can hear; because they are produced through binaural audio, the player shares the experience of these confusing and often hateful messages.

Monsterhearts incorporates a game move called Turn Someone On. When a player chooses to Turn Someone On, they invoke a sexual response in another player's character. The player may decide what form that sexual response takes, but it must be incorporated into their character's next action. Players are encouraged to use Turn Someone On regardless of gender, which means the rule can introduce explicit queer content into the game. However, even if the player uses Turn Someone On in a heterosexual context, the move still serves to challenge dominant notions of sexuality as fixed (the character is turned on regardless of gender and sexual orientation), predictable (the player does not know who will turn their character on in the future), and controllable (a player cannot deny their character's arousal).

In Thou Art But A Warrior, the protagonists are Muslim knights defending the Golden Age kingdoms of medieval Spain; as an intentional and historically appropriate reversal of Islamophobic tropes, the game positions Christians as monstrous invaders who seek to destroy the civilized world. Although the characters are meant to fight for their people, the game rules define a tragic arc for both the characters and the kingdom they seek to defend. When a knight behaves sympathetically to the infidel invaders, or fails to defend their own people, they may accumulate points toward Weariness and Discord. A knight who ends the game with a Weariness score of 4 either dies or converts to Christianity; these are mechanically equivalent, which suggests that they are equally disastrous outcomes for the character and their world. The 
dynamics of Weariness and Discord not only center the character's Muslim identity, but also deliberately decenter Christianity.

In both digital and non-digital systems we observe that rules can be used as ways of representing non-normative identity exploratively (e.g., as capacities for strength or as areas of difficulty or weakness). Acknowledging the interdependence of character representations, we see that rules and systems often operate on abstract representations. However, the structure of rules and the system can say something about non-normative identities beyond character representation. For example, the move Turn Someone On is not about how you represent the character's sexual desire as internal to the character, but is instead the way the character can take an action in the world. While both digital and nondigital games explore non-normative identities through mechanics such as new ability or added restriction, we see difference in how players interact with these systems. In digital games it is the system that does the processing and that must both define and adapt to the interactions, while in non-digital games the player may much more easily find themself in, or actively seek, corner cases where they can and may break the rules.

\section{DISCUSSION AND IMPLICATIONS}

Compared to the dominant strategy of locating difference in the player's avatar, the strategies discussed above allow the representation of more types of difference, including those that are not always visually marked (e.g., neurodiversity); those whose visual markers can vary or, when using supposedly prototypical visual markers, may reinforce stereotypes (e.g., Jewishness); and those that are only visible in certain circumstances (e.g., bisexuality). They also allow for the possibility of strategic introduction or integration of elements of difference within a game's content, mechanics, and rule-set. As suggested by the "embedded design" model of persuasive games (Kaufman et al. 2016b), deploying tactics such as delaying the disclosure of 
"otherness" in characters, interweaving elements of "otherness" alongside normative elements, and using more implicit and subtle means of communicating messages of diversity or difference can facilitate player embodiment of diverse characters and increase the positive impact of encounters with those characters as a result.

Of course, with the deployment of these approaches to depicting diversity and difference - particularly with a player base that itself is diverse in the identities, backgrounds and experiences represented - come a number of challenges which we must confront. First, the depiction of group marginalization may, for some players at least, unintentionally reinforce marginalization. For example, high-SES players who played a game simulating the daily life decisions of someone living under the poverty line were shown to emerge with less empathy and illusions of high agency in their views toward people in poverty (Roussos \& Dovidio 2016). On the one hand, one could argue that when the goal of avoiding stereotypical depictions dictates the use of coded, symbolic, or subtle representations, this may mean that diversity will only be explicitly recognized by players who belong to those groups. At the other extreme are examples of games that present stereotypes front-and-center to invite players to subvert them or treat them ironically (e.g., the tongue-in-cheek nature of stereotypes in Ladies \& Gentlemen or Cards Against Humanity). Inviting subversion in this way can be effective for players who come from a perspective of a deep understanding of bias, but what about players who might miss the point and use the game to reinforce stereotypes? Finally, it's important to anticipate the possibility of players confronting diversity or utilizing it in unintended and potentially problematic ways. For example, players may attempt to use difference instrumentally, either leveraging disadvantage or "victimhood" as persuasive devices to get their way in a game or, at the other extreme, casting difference as superhuman and exaggerating a dimension of difference for its effect (e.g., media depictions of people with disabilities have been shown to perpetuate both of these fallacies: Clogston 1990). In all of these ways, thinking deeply about the intersection of player identity and perspective, the 
points of difference tackled by a game, and the methods used to represent them, reveals the potential pitfalls, and also sets up the creative challenges in depicting diversity effectively.

Match diversity affordances to player needs. We discovered that different types of games use different techniques to represent diversity in games. However, we do not rank those different capacities in terms of absolute effectiveness. Rather, we consider that different capacities may be more appropriate for players with different needs around diversity. For example, many digital games constrain player input and are difficult to modify, implying that players must respond to diverse characters using options created by the game designers. For players who have little experience with a particular type of difference, or for players who may have negative stereotypes about that group, this approach may be helpful in modeling appropriate ways to react. However, players who personally experience a particular type of marginalization may want games that easily let them customize and express their own experiences, such as role-playing games. We note that experiencing marginalization along one axis (e.g. sexual orientation) does not mean that a person understands or experiences marginalization along other axes (e.g. immigration status), or that they are exposed to it in their daily lives. The same player may therefore benefit from exposure to a spectrum of diverse characters in a range of different games that use a variety of techniques to portray those differences. As we continue to develop a design language for the representation of diversity, we can more clearly identify the strengths and weaknesses of representation in individual games, and help match those games to the players who need them.

Draw strengths from the digital and non-digital realms. Although we have talked about games as digital or non-digital, this line is not as bright as it might appear. For example, Monsterhearts takes advantage of many of the affordances of the digital, even though its representation of queerness is rooted in the analog. Every physical game book comes with a digital PDF, which makes 
the rules searchable; the game can be played over video chat, using augmented tabletop tools to share game materials; and skins are distributed online. In the latter case, the strengths of the digital and non-digital work together to allow a deeper exploration of queerness. The Monsterhearts book provides explicit instructions on how players can create their own skins that represent different types of monstrous queerness, without the need for any special software or skill. However, they can also easily access skins made by others and share their own. Because players can explore skins shared digitally by other players across the global digital landscape, they can access unimaginable non-normative (or here, monstrous) identities and experiences that otherwise are inaccessible. Even though it happens outside of game sessions, this type of sharing and reflecting can be understood as a larger part of the game's engagement with diversity - and it is enabled by the game’s measured engagement with technology.

Design for conversation. While role-playing games are often described as a conversation (Alder 2012), we argue that other types of game design can also be conceptualized as conversation design (To et al. 2017). Board games, for example, often feature table talk (Xu et al. 2011). Even single-player digital games like the Dragon Age series foster larger cultural conversations, for example through media coverage, fan works, and discussion (Jenkins et al. 2013). The conversations that players are having in and around the game can be understood as locations for representing, surfacing, and reflecting on diversity. Considering the conversations that players are having before, during, and after play, can allow designers to identify opportunities for the player to confront difference. However, game designers must consider that those conversations are also opportunities for diversity and difference to be undermined, such as in the case of "ironic" jokes about racism, or would-be subversion of gender tropes that actually reinforces them.

Consider player diversity. In our analysis, we have located diversity in the game experience, but games are not simply sets 
of rules, objects in a box, or lines of code. They are experiences that are shared with other players, including before and after play. In other fields, there are interventions that seek to gather diverse groups of people to connect, including for learning (Kulkarni et al. 2015). Exposure to difference does not have to be entirely carried by within-game content; playing with people different from oneself, particularly when there are a range of experiences along different axes of marginalization, can also serve to increase exposure to diversity in games. While the composition of playgroups and game conversations may seem like it is outside the scope of game designers' remit, game designers have a great deal of influence over the ways that players connect. Three examples include the design of player matching systems, particularly for digital games but also including resources to find local playgroups for analog games; methods for controlling harassment of vulnerable subgroups; and the design of cover art, descriptive text, and other game materials that players use to determine whether or not they are a part of the game's target audience.

\section{CONCLUSION}

In this paper, we have explored a range of ways that marginalized or non-normative experiences can be represented in game characters in ways that go beyond customization of avatars. Additionally, we extend our analysis to understand the diversityrelated affordances of digital and non-digital games. Finally, we derive four implications for game design for diversity: match diversity affordances to player needs; draw strengths from both digital and non-digital realms; design for conversation; and consider player diversity.

Based on this work, we believe that we should be looking at diversity in games as an end-to-end process, starting when the platform and audience for the game, as well as its funding and distribution models, are being considered, incorporating both before and after-play activities as well as what happens during 
game sessions, and continuing through to the way that games are used in players' lives (e.g. the conversations they are having about the game and who they choose to play with). This approach gives us a lens to bring together research on games and diversity across a range of different fields, including close critical reading of games, conversational analysis from play-groups, economic analysis of distribution and funding models, and analysis of fanworks and other media.

An end-to-end process approach challenges us to consider design issues not typically incorporated into diversity in games. For example, how do we understand the lifespan of games as a medium for representing diversity in a changing society? Groups' experiences and social positions change over time; we need to consider the cultural assumptions that travel along with games into a changed future world. Furthermore, how do we design diverse representation in games when we're unsure how long they will be relevant? While the Dragon Age franchise may persist, we may lose the opportunity to play those games due to changes in hardware in ten years, let alone fifty or a hundred. In contrast, some non-digital games have been played for hundreds or thousands of years. How can we design games to represent diversity when they may exist in a future with identity dynamics we cannot even imagine? As scholars and designers, these are the questions we ask ourselves, and by challenging normative assumptions found in games and game culture, we might one day answer them.

\section{ACKNOWLEDGMENTS}

We would like to thank the experts and colleagues who contributed to our list of games. 


\section{BIBLIOGRAPHY}

Alder, A. 2012. Monsterhearts. [Tabletop] Buried Without Ceremony.

Altice, N., 2014. The Playing Card Platform. Analog Game Studies.

Bailey, M., 2010. They aren’t talking about me...'. Crunk Feminist Collective, 18.

Baldwin, M. W. (1992). Relational schemas and the processing of social information. Psychological Bulletin, 112(3).

BioWare. (2009, 2011, 2014). Dragon Age. [PC Computer, Console] Electronic Arts.

Bizzocchi, J. and Tanenbaum, J., 2011, January. Well read: Applying close reading techniques to gameplay experiences. In Well played 3.0 (pp. 262-290). ETC Press.

Bogost, I. and Montfort, N., 2007. New Media as Material Constraint. An Introduction to Platform Studies'. THINKING AT THE INTERFACE.

Chance, T.R. and Little, N. 2014. Heroes Wanted. [Tabletop] Action Phase Games.

Clogston, J. S. (1990). Disability coverage in 16 newspapers. Louisville, KY: Advocado.

Cole, A., Shaw, A., Zammit, J., 2017. Representations of Queer Identity in Games from 2013-2015. Transactions of the Digital Games Research Association.

Crenshaw, Kimberle, 1989. Demarginalizing the intersection of race and sex: A black feminist critique of antidiscrimination 
doctrine, feminist theory and antiracist politics. U. Chi. Legal F.: 139.

Dasgupta, N. and Greenwald, A.G., 2001. On the malleability of automatic attitudes: combating automatic prejudice with images of admired and disliked individuals. Journal of Personality and Social Psychology,81(5).

Davis, M.H., Conklin, L., Smith, A. and Luce, C., 1996. Effect of perspective taking on the cognitive representation of persons: a merging of self and other. Journal of Personality and Social Psychology.

Dillon, J., Dranove, D., Halpern, E., Hantoot, B., Munk, D., Pinsof, D., Temkin, M. and Weinstein, E., 2009. Cards Against Humanity. [Tabletop] Self-published.

Ellingboe, J. B. 2007. Steal Away Jordan. [Tabletop] Stone Baby Games.

Fernández-Vara, C., 2014. Introduction to game analysis. Routledge.

Galinsky, A.D. and Moskowitz, G.B., 2000. Perspective-taking: decreasing stereotype expression, stereotype accessibility, and ingroup favoritism. Journal of personality and social psychology, 78(4).

Game Grumps. 2017. Dream Daddy. [PC Computer]. Game Grumps.

Hanako Games. 2012. Long Live the Queen. [PC Computer].

Hoffner, C., 1996. Children's wishful identification and parasocial interaction with favorite television characters. Journal of Broadcasting \& Electronic Media, 40 (3). 
Hogg, Michael A., Deborah J. Terry, and Katherine M. White, 1995. "A tale of two theories: A critical comparison of identity theory with social identity theory." Social psychology quarterly: 255-269.

Jagose, A., 1996. Queer theory: An introduction. NYU Press.

Jenkins, H., Ford, S. and Green, J., 2013. Spreadable media: Creating value and meaning in a networked culture. NYU press.

Jiow, H.J. and Lim, S.S., 2012. The evolution of video game affordances and implications for parental mediation. Bulletin of Science, Technology \& Society, 32(6).

Kafai, Y.B., Fields, D.A. and Cook, M.S., 2010. Your second selves: Player-designed avatars. Games and culture, 5(1).

Kaufman, G.F., Flanagan, M. and Belman, J., 2016a. Playing the System: Comparing the Efficacy and Impact of Digital and NonDigital Versions of a Collaborative Strategy Game. In DiGRA/ FDG.

Kaufman, G., Flanagan, M., and Seidman, M. 2016b. Creating stealth game interventions for attitude and behavior change: An" Embedded Design” model. Transactions of the Digital Games Research Association, 2(3).

Kaufman, G.F. and Libby, L.K., 2012. Changing beliefs and behavior through experience-taking. Journal of personality and social psychology,103(1).

Khaled, R., 2014. Questions over answers: Reflective game design. Playful Subversion of Technoculture.

Kidd, D.C. and Castano, E., 2013. Reading literary fiction improves theory of mind. Science, 342(6156).

KOSMOS. (2012). Legends of Andor. [Tabletop] 
Kulkarni, C., Cambre, J., Kotturi, Y., Bernstein, M.S. and Klemmer, S.R., 2015, February. Talkabout: Making distance matter with small groups in massive classes. In Proceedings of the 18th ACM Conference on Computer Supported Cooperative Work \& Social Computing. ACM.

Lamy, L. 2013. Ladies \& Gentlemen. [Tabletop] Asmodee.

Mar, R.A., Oatley, K., Hirsh, J., dela Paz, J. and Peterson, J.B., 2006. Bookworms versus nerds: Exposure to fiction versus nonfiction, divergent associations with social ability, and the simulation of fictional social worlds. Journal of Research in Personality, 40(5).

Markus, H., \& Wurf, E. 1987. The dynamic self-concept: A social psychological perspective. Annual Review of Psychology, 38(1).

Marx, D.M. and Roman, J.S., 2002. Female role models: Protecting women's math test performance. Personality and Social Psychology Bulletin,28(9).

McArthur, V., Teather, R.J. and Jenson, J., 2015, October. The avatar affordances framework: mapping affordances and design trends in character creation interfaces. In Proceedings of the 2015 Annual Symposium on Computer-Human Interaction in Play(pp. 231-240). ACM.

McCall, Leslie. "The complexity of intersectionality." Signs: Journal of women in culture and society30.3 (2005).

Murray, J.H., 2011. Inventing the medium: principles of interaction design as a cultural practice. Mit Press.

Plaid Hat Games. 2016. Dead of Winter: The Long Night.[Tabletop]

Portal Games. (2012). Robinson Crusoe: Adventures on the Cursed Island.[Tabletop] 
Roussos, G., and Dovidio, J. F., 2016. Playing below the poverty line: Investigating an online game as a way to reduce prejudice toward the poor. Cyberpsychology: Journal of Psychosocial Research on Cyberspace, 10(2).

Ruberg, B. and Shaw, A., 2017. Queer game studies. University of Minnesota Press.

Sellen, A.J. and Harper, R.H., 2003. The myth of the paperless office. MIT press.

Shaw, A., 2009. Putting the gay in games: Cultural production and GLBT content in video games. Games and Culture, 4(3).

Shaw, A., 2010. Identity, identification, and media representation in video game play: An audience reception study(Doctoral dissertation, University of Pennsylvania).

Shaw, A., 2012. Do you identify as a gamer? Gender, race, sexuality, and gamer identity. new media \& society, 14(1).

Strauss, A. and Corbin, J., 1998. Basics of qualitative research techniques. Sage publications.

Suits, B., 2014. The Grasshopper-: Games, Life and Utopia. Broadview Press.

To, A., Kaufman, G., Hammer, J., 2017. Scaffolding Conversations through the Design and Implementation of Board Games. In DiGRA Conference.

Toma, E. (2010). Powerful elderly characters in video games: Flemeth of Dragon Age. Journal of Comparative Research in Anthropology and Sociology, 1(1), 163-174.

Turkay, S. and Adinolf, S., 2010. Free to be me: a survey study on customization with World of Warcraft and City Of Heroes/Villains players. Procedia-Social and Behavioral Sciences, 2(2). 
64 Sian Beavers \& Darshana Jayemanne

Turkington, M. 2016. Against the Grain. [Tabletop]. Unruly Designs.

Warne, P.D. Larklamp. 2017. [Tabletop] Lumo Amuzo.

Williams, D., Martins, N., Consalvo, M. and Ivory, J.D., 2009. The virtual census: Representations of gender, race and age in video games. New Media \& Society, 11(5).

Wiley, M. G., 1991. Gender, work, and stress: The potential impact of role-identity salience and commitment. The Sociological Quarterly,32(4).

Xu, Y., Barba, E., Radu, I., Gandy, M. and MacIntyre, B., 2011. Chores Are Fun: Understanding Social Play in Board Games for Digital Tabletop Game Design. In DiGRA Conference. 


\section{APPENDIXA}

\begin{tabular}{|l|l|}
\hline Non-Digital Game Titles & Digital Game Titles \\
\hline Android:Netrunner & Bully \\
\hline Against the Grain & $\begin{array}{l}\text { Dragon Age series (i.e., Dragon Age: Origins, } \\
\text { Dragon Age II, Dragon Age: Inquisition) }\end{array}$ \\
\hline Arctic Scavengers & Dream Daddy \\
\hline $\begin{array}{l}\text { Arkham Horror (or Eldritch Horror or } \\
\text { Mansions of Madness) }\end{array}$ & Gone Home \\
\hline Ashes: Rise of the Phoenixborn & Grand Theft Auto \\
\hline Dead of Winter: The Long Night & Hellblade: Semua's Sacrifice \\
\hline Descent & Mass Effect: Andromeda \\
\hline Dogs in the Vineyard & NBA Street Volume 2 \\
\hline Fog of Love & Never Alone \\
\hline Funny Friends & Night in the Woods \\
\hline Heroes Wanted & Saint's Row 3 \\
\hline Magic the Gathering & Sim City \\
\hline Misspent Youth & Starcraft: Broodwar \\
\hline Monsterhearts & Stardew Valley \\
\hline Pathfinder & Tacoma \\
\hline Sentinels of the Multiverse & True Crime: Streets of LA \\
\hline Steal Away Jordan & \\
\hline The Curse & \\
\hline The Magicians & \\
\hline The Watch & \\
\hline The \#feminism anthology & \\
\hline Thou Art But a Warrior & \\
\hline Vampire the Masquerade & \\
\hline 14 Days & \\
\hline Sal & \\
\hline
\end{tabular}

\section{Game Inventory}

